\section{Strategy for the Development of Vaccines Against Chagas Disease}

\author{
Yanfen $\mathrm{Ma}^{1}$, Louis M Weiss ${ }^{1,2}$ and Huan Huang ${ }^{1 *}$ \\ ${ }^{1}$ Department of Pathology, Albert Einstein College of Medicine Jack and \\ Pearl Resnick Campus, 1300 Morris Park Avenue, Bronx, NY 10461, USA \\ ${ }^{2}$ Department of Medicine, Albert Einstein College of Medicine Jack and \\ Pearl Resnick Campus, 1300 Morris Park Avenue, Bronx, NY 10461, USA
}

\begin{abstract}
Chagas disease caused by Trypanosoma cruzi, is a major public health issue, particularly in the Americas. There are approximately 10 million infected people and more than ten thousand annual deaths due to this parasite. T. cruzi infection usually results in parasite persistence in the infected host. The available therapeutic agents have limited efficacy in these chronic infections and also have significant side effects which limit their usefulness. There are limitations to the innate and acquired immune responses to the infection in a non-immunized host. Currently, no vaccine is available for protecting against $T$. cruzi infection, despite considerable research in this area. A safe, effective and reliable prophylactic vaccine should significantly reduce the threat of $T$. cruzi infections and prevent Chagas disease. In addition, an efficient therapeutic vaccine should improve the outcome of treatments. Research indicates that the development of vaccines against $T$. cruzi infection is feasible and highly significant. This article reviews efforts to develop vaccines against Chagas disease. In addition, the authors discuss a new strategy to develop safe and effective vaccines against $T$. cruzi infection.
\end{abstract}

\section{Introduction}

Chagas disease or American trypanosomiasis, is a neglected tropical disease caused by the protozoan Trypanosoma cruzi (T. cruzi) [1]. Currently, this disease affects about 10 million people in endemic Latin American country and is becoming a significant health issue globally due to immigration to many developed countries [1]. Every year, more than forty thousand new cases occur in endemic areas and about fourteen thousand infants are born with congenital infection [2-3]. Chagas disease causes more than ten thousand deaths and results in over $\$ 7$ billion in lost productivity and health care costs annually [4].

*Corresponding author: Huan Huang, Department of Pathology, Albert Einstein College of Medicine Jack and Pearl Resnick Campus, 1300 Morris Park Avenue, Bronx, NY 10461, USA, Tel: +1 7184302143; Fax: +1 7184308543; E-mail: Huan.huang@einstein.yu.edu

Citation: Ma Y, Weiss LM, Huang H (2015) Strategy for the Development of Vaccines Against Chagas Disease. J Vaccines Res Vaccin 1: 001.

Received: September 14, 2015; Accepted: October 29, 2015; Published: November 18, 2015
The major mode of transmission of T. cruzi in endemic countries is through infected insect vectors called triatomine or Reduviid bugs (i.e., Rhodinius, and Panstrongylus) [2]. These infected bugs usually take blood meals from people at night and then defecate while feeding. Their feces contain metacyclic trypomastigotes. These metacyclic trypomastigotes enter the host through the bite wound or through intact mucosal membranes. Inside the host, the trypomastigotes invade cells, where they differentiate into intracellular amastigotes. Amastigotes multiply by binary fission and differentiate into trypomastigotes, which are then released into the circulation as bloodstream trypomastigotes. When the bugs take blood meals containing trypomastigotes from infected people or animals, the parasites transform to a dividing form called epimastigotes in the insect midgut. Epimastigotes then differentiate into infective metacyclic trypomastigotes in the insect hindgut. T. cruzi can also be transmitted through blood transfusions, organ transplantation, oral intake of contaminated foods [5], mother-tochild and laboratory accidents [6-7]. T. cruzi infection can occur in more than 150 other animals [2].

In the early stage of $T$. cruzi infection, many infected patients are asymptomatic or have mild symptoms such as fever, swollen lymph nodes, headaches, or local swelling at the site of the bite [7]. After 2 to 3 months, individuals enter the chronic stage of T. cruzi infection, where the majority of individuals remain asymptomatic while about $30 \%$ develop chronic cardiomyopathy, leading to heart failure or sudden death and about $10 \%$ develop an enlarged esophagus or enlarged colon [7]. Current pharmacological treatments are depended on benznidazole or nifurtimox [8]. Antiparasitic treatment is effective if given very early in the course of infection, but has limited effect on chronic infection. Since many patients are asymptomatic after infection, these patients usually miss the window for early treatment. Resistance to these drugs has been reported in treated patients [9]. Recently, a prospective, multicenter, randomized study has been completed. Trypanocidal therapy with benznidazole in patients with established Chagasic cardiomyopathy significantly reduced serum parasite detection but did not significantly reduce cardiac clinical deterioration through 5 years of follow-up [10].

Currently, prevention focuses on vector control by decreasing the numbers of the triatomine bugs that transmit the infection using insecticides and by preventing the contact of these bugs with humans using mosquito nets and improving housing conditions. These measures significantly reduce disease incidence and prevalence in endemic countries [11]. However, in spite of the vector control interventions, a number of triatomine bugs have proven to be difficult to control and a low level of transmission to humans continues. Therefore, a safe, effective and reliable vaccine should significantly reduce the threat of T. cruzi infections and prevent the development of Chagas disease. Studies highlight the significant cost-effectiveness of prophylactic vaccine for humans [12]. A therapeutic vaccine that boosts immune responses to combat chronic infection may greatly improve the prognosis for Chagasic patients by increasing treatment efficacy, reducing its duration and cost, or delaying disease progression to advanced stages and heart failure. In addition, an effective veterinary vaccine should provide control of the domestic 
reservoir of T. cruzi (dogs) in specific geographical regions, contributing to the effectiveness of Chagas disease control programs.

Currently, there is no suitable vaccine against Chagas disease despite considerable research in this area. In this article, we review efforts to develop vaccines against T. cruzi infection. Moreover, we also discuss some new techniques and novel strategies to design vaccines protecting against Chagas disease based on work in our laboratory group.

\section{Protective Immunity Against Trypanosoma Cruzi Infection}

Our knowledge of protective immunity against T. cruzi infection is still incomplete. We do not understand the mechanisms why the immune system fails to totally eradicate the parasite once T. cruzi infection is established. An improved understanding of the interactions between host immune response and T. cruzi infection will provide rational rules for vaccine design and development. In recent years, the research community has made progress in understanding both innate and adaptive immune responses against $T$. cruzi infection.

One striking feature of $T$. cruzi infection is that $T$. cruzi infection usually results in parasite persistence in the infected host. This phenomenon indicates that there are limitations to the innate and acquired immune responses to the infection. Pattern Recognition Receptors (PRR) which recognize Pathogen-Associated Molecular Patterns (PAMPs) such as Toll-Like Receptors (TLRs) 2, 4, 7, and 9, Nucleotide-binding Oligomerization Domain-like receptor (NOD) 1 have been shown to participate in $T$. cruzi recognition by macrophages and dendritic cells [13-18]. In the initial period after infection (days 7 to 10 following infection) innate responses play a key role in containing parasitemia, through the activation of innate immune response pathways leading to the activation of NFK-B/AP-1 and the productions of microbicidal mediators such as Reactive Nitrogen Intermediates (RNI) and Reactive Oxygen Species (ROS). The production of these microbicidal mediators are enhanced by the action of proinflammatory cytokines (IL-12, TNF- $\alpha$, and IFN- $\gamma$ ) released by macrophages, Natural Killer (NK), and $\gamma \delta$ T cells [19-20].

Following this innate response, acquired immunity mediated by the T-helper 1 (Th1) cell response plays a key role in controlling parasitemia and host survival. IFN- $\gamma$ secreted by Th $1 \mathrm{CD}^{+}$cells induces the activation of phagocytic cells for parasite killing. Th1 lymphocytes also stimulate the appropriate production of antibodies by $\mathrm{B}$ cells and the activation of cytotoxic $\mathrm{CD} 8^{+} \mathrm{T}$ cells. However, the development of adaptive immunity to $T$. cruzi infection is relatively slow with delayed development of $T$. cruzi-specific $\mathrm{CD}^{+} \mathrm{T}$ effector cells [21] which may be explained by diverse factors, including the postulated poor PAMP activity of T. cruzi [22]. T. cruzi is able to suppress the immune system through the release of several molecules and can promote IL-10 production by dendritic cells favoring its persistence [23]. T. cruzi also induces death signaling molecules such as Fas on T. cruzi specific CD8 ${ }^{+} \mathrm{T}$ cells [24] as well as PD-1 and PD-L1 on $\mathrm{CD}^{+}$and $\mathrm{CD} 8^{+} \mathrm{T}$ cells. The persistent infection causes the senescence of $\mathrm{CD}^{+} \mathrm{T}$ cells [25] and exhaustion of $\mathrm{CD}^{+} \mathrm{T}$ cells [26-27]. Therefore, a prophylactic vaccine should be aiming at early recognition and killing of $T$. cruzi and a therapeutic vaccine should be aiming at counteracting the immune suppressive mechanisms mediated by the parasites and boosting the host Th1 immune response for parasite killings.

\section{Subunit Experimental Vaccines}

The early efforts in developing subunit vaccines against $T$. cruzi tried to identify molecules that provided protective immunity against T. cruzi infection using sub cellular fractions of T. cruzi or purification of $T$. cruzi proteins as experimental vaccines. In sub cellular fractions from epimastigotes (Tulahuén strain), the flagellar fraction gave a partial protection in a challenge experiment [28-29]. The Paraflagellar rod proteins (PAR) purified from epimastigotes were shown to protect mice against a lethal challenge. The protective immunity in mice induced by PAR with adjuvants was associated Th1 response and cell mediated responses induced by PAR was more important than humoral responses in immunoprotection against T. cruzi infection [30- 31]. Two cell surface glycoproteins $(90 \mathrm{kDa}$ and $72 \mathrm{kDa})$ were found to have the ability to protect mice against an acute lethal infection from both bloodstream and metacyclic trypomastigotes [32]. Afterwards, several T. cruzi proteins were purified by different methods and these molecules demonstrated some levels of protective activities against T. cruzi lethal infection [33-36]. The role of IFN- $\gamma$ in controlling parasite replications and against lethal infection in mice was reported with a T. cruzi soluble extract antigen treatment in mice [37].

The application of native antigens purified from T. cruzi as experimental vaccines has limitations because it is difficult to obtain sufficient amounts of these antigens to perform the studies. With the availability of $T$. cruzi genome and the modern biotechnology for protein expression, many T. cruzi genes can be used to produce a large amount of recombinant proteins which can be further tested for the ability to protect animals against T. cruzi infection.

Recombinant proteins of Cytoplasmic Repetitive Antigen (CRA), and Flagellar Repetitive Antigen (FRA) were used to immunize Balb/c mice and the levels of IgG1, IgG2a, IgG2b and IgG3 isotypes were analyzed. CRA-immunized mice produced high levels of all IgG isotypes, mainly IgG3 and IgG1. FRA-immunization elicited only high levels of IgG1 [38]. With co-adsorbed to alum and recombinant murine IL-12, recombinant Paraflagellar Rod proteins (PFR), which are conserved among seven highly diverse strain of T. cruzi, induced protective immunity against $T$. cruzi [39].

Immunization with the recombinant trans-sialidase generated a Th2-type immune response while using a plasmid, p154/13 which encodes the same T. cruzi trans- sialidase, induced a Th1-type immune response in mice. Simultaneous administration of both p154/13 and recombinant T. cruzi trans-sialidase protein also led to a predominant type 2 immune response. Two priming doses of p154/13 harboring the gene followed by booster injections with recombinant T. cruzi trans-sialidase protein significantly improved specific type 1 immune response, as revealed by a drastic reduction of the serum IgG1/ IgG2a ratio and by an increase in the in vitro IFN- $\gamma$ secretion by CD4 T cells. However, mice immunized with the DNA-priming protein-boosting regimen did not have better survival rate with the challenge as compared to mice with DNA alone, indicating that the boost did not contribute protective immunity against T. cruzi infection [40]. Different regions of recombinant proteins from a major T. cruzi cystein proteinase, cruzipain, were used to investigate the protective immunity in mice. The $\mathrm{N}$ - terminal domain as an immunogen with CpG-ODN as an adjuvant was able to direct the host with better protective immunity [41].

Recombinant protein vaccines have some disadvantages against T. cruzi infection. The protection is usually insufficient because 
T. cruzi can still establish persistent infection in the immunized animals. T. cruzi strains are highly diverse and it is difficult to have a protein vaccine that can confer broad protections cross different strains. In addition, protein vaccines are more suitable for inducing antibody responses and are not efficient in eliciting the cell-mediated immunity that is essential for controlling the intracellular stage of a pathogen. In their favor, recombinant protein vaccines are safe and have been licensed for hepatitis B and human papillomavirus. With some types of adjuvant, some T. cruzi protein vaccines have been demonstrated to induce a degree of protective immunity. Even with a moderate protection, immunization with a protein vaccine may still change the outcome of morbidity and mortality of T. cruzi infection. Recombinant protein vaccines are more feasible for the population in poor areas to gain some immunizations. Therefore, recombinant protein vaccines Tc24 and TSA- 1 formulated on alum together with the Toll-like receptor 4 agonist, E6020, are being tested in patients [42]. Techniques to produce large amount of Tc24 recombinant proteins in Escherichia coli or in yeast were reported and the recombinant proteins were tested as experimental vaccines that demonstrated partial protective efficacy in murine models [43].

Another approach termed DNA vaccines has also been developed, using a vector to express the antigen(s) in hosts [44]. The direct introduction of a plasmid containing a DNA sequence encoding T. cruzi antigen(s) into appropriate tissues has been tested. An immune response relies on the in situ production of the target antigen. This approach offers a number of potential advantages over traditional approaches, including the stimulation of both B- and $\mathrm{T}$-cell responses and improving long-term persistence of immunogen. Moreover, these types of vaccines are easy to produce, store and ship and therefore, the approach is cost effective. However, there are disadvantages of using this approach. It is limited to protein antigens with the possibility that tolerance to the antigens by the hosts may occur because of the persistence of immunogen. In addition, there is a risk of affecting host genes controlling cell growth, leading to the development of malignancy. Autoimmunity is still considered as one of the factors contributing to Chagas disease and the persistent presentation of T. cruzi antigens in host tissues may be a concern for possible development of pathology. In spite of these concerns, experimental DNA vaccines against Chagas disease are progressing.

Murine immunization with DNA vaccine using a chimeric gene of T. cruzi kinetoplastid-specific KMP11 Protein and Heat-Shock Protein-70 (HSP70), but not the KMP11 gene alone, induced both IgG2 long-lasting humoral immune response against KMP11 protein and activation of $\mathrm{CD}^{+}$cytotoxic $\mathrm{T}$ lymphocytes specific for two KMP11 peptides. Mice immunized with the chimeric gene also gained protection against parasite challenge. This study indicates that immunization of animals with antigens fused to heat shock proteins in the absence of an adjuvant elicits antigen- specific immune responses [45]. In addition, HSP70 alone or fused to the KMP11 antigen, as well as a HSP70 fragment, were capable of stimulating the maturation of murine dendritic cells, suggesting that HSP70 may be used as a candidate or as an adjuvant for vaccination [46]. The immunization of mice with the DNA vectors containing the Paraflagellar Rod proteins (PFRs) genes or PFRs-HSP70 fused genes induced high level of IgG2 anti-PFRs. The immunization with the PFR2-HSP70 fused genes triggered a Th1 response profile and protection against $T$. cruzi infection [47]. T. cruzi HSP70 was subsequently studied for its role in inducing immunity against T. cruzi infection [48-50]. A cDNA clone that codes for an amastigote-Specific Surface Protein (TcSSP4) was used as a candidate to develop a DNA vaccine. Mice immunized with a eukaryotic expression plasmid containing the TcSSP4 cDNA were able to reduce the acute phase of infection and induce a Th1 response, resulting in a better immune protection than that of using a recombinant protein vaccine from the same gene [51]. Similarly, the TcSP gene with DNA-based immunization provided a better protective immunity than that of using recombinant protein [52]. Both DNA vaccines were then further tested in a canine model of Chagas disease. Immunizations with DNA vaccines coding both genes in Beagle dogs reduced the severity of clinical signs of acute infection including electrocardiographic abnormalities [53]. Immunizations with both genes also induced IgG2 immunoglobulin's and IFN- $\gamma$ productions as well as lymph proliferation, indicating cell mediated immunity. In chronic stage, immunized dogs with DNA vaccines coding both genes challenged with T. cruzi still developed myocarditis with a partial protection from the experimental vaccines [54].

The cDNAs for TcG2 and TcG4, which code for membrane-associated GPI proteins of $T$. cruzi, were cloned into eukaryotic expression plasmid pCDNA3.1 to make the experimental vaccine, TcVac3. A DNA-prime/MVA-boost approach in C57BL/6 mice against T. cruzi challenge was reported. Vaccination with TcVac3 elicited the production of lytic antibodies against T. cruzi and antigen- and parasite-specific CD4/CD8 $\mathrm{T}$ cell proliferation. The $\mathrm{CD}^{+} \mathrm{T}$ cells were cytolytic effector cells with predominantly IFN $-\gamma+$. In the chronic stage of infection, immunized/infected mice exhibited a significant decline in IFN $-\gamma^{+} \mathrm{CD} 8^{+} \mathrm{T}$ cells with a predominance of immunoregulatory $\mathrm{IL}-10^{+} / \mathrm{CD} 4^{+} \mathrm{T}$ and $\mathrm{IL} 10^{+} / \mathrm{CD} 8^{+} \mathrm{T}$ cells, indicating a shift to type 2 cytokine and $\mathrm{T}$ cell response which reduce the development of cardiomyopathy [55]. Subsequently, TcVac3 was tested as a therapeutic vaccine in mice infected with $T$. cruzi and demonstrated some therapeutic efficacy in reducing parasite burden and cardiac damages in chronic stage [56].

DNA prime/protein boost vaccine ( $\mathrm{TcVac} 2)$ based on a eukaryotic expression plasmid pCDNA3.1 coding for the cDNAs for TcG1, $\mathrm{TcG} 2$, and TcG4 was tested in C57BL/6 mice. Immunized mice demonstrated a significant reduction of parasite burden and myocarditis with the production of lytic antibodies against $T$. cruzi and generation of type $1 \mathrm{CD} 8^{+} \mathrm{T}$ cells. The TcVac2 vaccine reduced chronic myocarditis due to the anti-proliferative and anti-inflammatory responses of macrophages [57].

A fragment of T. cruzi Amastigote Surface Protein-2 (ASP-2) was cloned into the backbone of Yellow Fever (YF) 17D virus as an experimental DNA vaccine against T. cruzi. YF 17D virus has been established as a human vaccine and therefore, using this system to express T. cruzi antigen(s) may facilitate the development of T. cruzi DNA vaccine for human. A/J mice were immunized subcutaneously with two doses of YF 17D recombinant virus and four weeks after the last dose they were challenged with T. cruzi. Mice immunized with recombinant YF 17D viruses expressing T. cruzi antigens resulted in a reduction in peak parasitemia, an increase in IFN- $\gamma$ - secreting splenocytes as well as a reduction in mortality in challenged mice [58].

DNA vaccines based on the pcDNA3.1 plasmid vector and encoding for TSA-1 and Tc24 T. cruzi antigens was tested in mongrel dogs. As a preventive vaccine, mongrel dogs were immunized with two doses of $500 \mathrm{~g}$ of DNA vaccine with aluminum phosphate as an adjuvant intramuscularly and infected with SylvioX10/4 strain of T. cruzi two weeks after the second vaccine dose. As a therapeutic 
vaccine, dogs was infected first and then treated with the vaccine. Both approaches of vaccinations did not improve survival rate in dog with $T$. cruzi challenge, indicating that the protective or therapeutic effects by the DNA vaccine were not sufficient. The effect of vaccination on the immune response was weak because it did not observe the induction of a humoral response although an increased level of IFN- $\gamma$ in immunized dogs was detected. Parasitemia, cardiac inflammation and cardiac parasite burden were reduced by preventive or therapeutic vaccine treatment [59], indicating that the DNA vaccination may be used to reduce $T$. cruzi transmission as veterinary vaccine.

Heterologous prime-boost vaccination using plasmid DNA followed by replication-defective adenovirus vector generates a large number of specific $\mathrm{CD} 8^{+} \mathrm{T}$ effect or memory cells that provide long-term immunity against a variety of pathogens. Plasmid pIgSPclone9 and human replication-deficient adenovirus type 5 expressing the amastigote surface protein, ASP-2 gene were generated and used to immunize mice. After T. cruzi challenge in the immunized mice, these mice showed the increase in the frequency of specific $\mathrm{CD}^{+} \mathrm{T}$ cells and these specific $\mathrm{CD} 8^{+} \mathrm{T}$ cells were capable of producing simultaneously the antiphrastic mediators IFN $-\gamma$ and TNF. Mice immunized with this $T$. cruzi virus vaccine had a significant increase in survival rate with T. cruzi challenge [60].

Attenuated Salmonella carrying plasmids coding for a T. cruzi cysteine protease, cruzipain, a T. cruzi -released protein related to thiol-disulfide oxidoreductase family, Tc52 and a $24 \mathrm{kDa}$ T. cruzi flagellar calcium-binding protein, Tc24 were generated and these bacterial strains bearing the DNA vaccines were administrated to mice orally. Immunization with either individual T. cruzi antigen or triple-antigen demonstrated lytic antibodies against trypomastigotes. Mice with the triple-antigen vaccination demonstrated better protection against T. cruzi infection [61]. Recently, it was reported that co-administration of cruzipain and GM-CSF DNAs as a new immunotherapeutic vaccine against Trypanosoma cruzi infection by intramuscular administration or by attenuated Salmonella. Both approaches showed some efficacy in preventing tissue damages in mice [62].

Replication-defective human Type 5 recombinant adenoviruses carrying sequences of amastigote surface protein- 2 and trans-sialidase T. cruzi antigens were used to immunize mice. Both vaccines were tested as prophylactic and therapeutic vaccines. Prophylactic immunization with both vaccines induced antibodies and specific cytotoxic and Interferon (IFN) $\gamma$-producing $\mathrm{CD}^{+} \mathrm{T}$-cells, reduced acute heart parasitism and electrical abnormalities in the chronic phase. Therapeutic vaccination with both vaccines increased survival and reduced electrical abnormalities [63]. A DNA vaccine termed TcVac4, which includes pcDNA3.1 encoding TcG1, TcG2, and TcG4 antigens plus IL-12- and GM-CSF-encoding plasmids, and glutaraldehyde-inactivated $T$. rangeli epimastigotes were tested in mongrel dogs. Dogs were immunized with two-doses of DNA vaccine followed by two doses of fixed parasites and then challenged with highly pathogenic T. cruzi. TcVac4 induced a strong IgG response which was expanded post-infection. Blood parasitemia, tissue parasite burden, electrocardiographic and histological abnormalities were lower in DNA-prime $(\mathrm{TcVac} 4) / T$. rangeli-boost dogs as compared to dogs given $T$. rangeli or empty plasmid DNA only [64].

In summary DNA vaccines have demonstrated some protection against T. cruzi infection in animals; however, the data from dog models indicate that the immune protection from current DNA vaccines is modest. With improvements in T. cruzi antigen selection and in expression system constructions, it is possible that DNA vaccine against Chagas disease may eventually become clinically feasible.

\section{Attenuated Trypanosoma Cruzi Experimental Vaccines}

Early efforts in developing vaccines against T. cruzi used heat-killed parasites. Unfortunately, these killed parasites did not elicit protective immunity [28]. A general principle for vaccines is that the more similar a vaccine is to the natural disease, the better the protective immunity that is seen. The success of live-attenuated microorganisms as vaccines against yellow fever, smallpox, measles, rubella, tuberculosis, and other pathogens supports this principle. The efficacy of these vaccines depends on the use of live naturally attenuated organisms and a truly self-limited infection is necessary to attain a strong and long lasting protection. Recently, Plasmodium was genetically attenuated and tested as a vaccine and this approach has been successful with this pathogen [65]. In T. cruzi, several naturally attenuated strains have been used in immunization-infection experiments in animal models and have provided strong protection [66-67]. In addition, single or double gene deletion to remove virulence or metabolic factors has been used to attenuate parasites and obtain attenuated strains of T. cruzi. A range of these genetically altered parasites has been evaluated as experimental vaccines.

One of the two alleles of the calmodulin-ubiquitin gene was disrupted in Tulahuen strain and the mutant strain demonstrated much lower parasitemia in mice as compared to the wild type. A clone of mutant epimastigotes was used to immunize mice and then challenge with wild type Tulahuen strain. The inoculation of mutant parasites in mice demonstrated a strong protection [68].

The deletion of both LYT1 alleles in the CL Brenner strain was achieved and a null mutant T. cruzi clone (L16) that showed an attenuated phenotype in culture and in mice. L16 blood counts were almost undetectable but blood-based PCRs indicated the presence of latent and persistent infection during all of the study period. The recovered parasites from infected mice showed that the parasites were lacking the LYT1 gene and still bearing the antibiotic resistance genes indicating the stability of the genetic manipulation. Inoculation with L16 provided a strong protection against a virulent challenge with a cogenic strain [69].

A targeted deletion of the gp72 gene in Y strain was performed and infection with this mutant in highly susceptible BALB suckling mice and immunodeficient athymic mice did not detect blood parasitemia. Only hemocultures from athymic mice in acute infection were able to isolate the mutant parasites. Inoculation with the mutant parasites in mice was able to induce long lasting protection against a subsequent infection by virulent $T$. cruzi [70].

To test the possibility of using attenuated T. cruzi for oral vaccination, $\mathrm{C} 57 \mathrm{BL} / 6$ mice were given mutant parasites lacking enoyl co-A hydratase gene orally. Oral inoculation of T. cruzi established infection in muscles and induced a robust $\mathrm{CD}^{+} \mathrm{T}$ cell response. Moreover, oral vaccination with the attenuated parasites provided protection against wild type $T$. cruzi challenge. Therefore, it is possible to develop live parasite oral vaccines, especially veterinary vaccine for the immunization of reservoir hosts [71]. Monoallelic mutant parasites for the $d h f r$-ts gene in both a naturally attenuated 
TCC T. cruzi strain and Tulahuen strain were generated. The mutant Tulahuen strain showed significant attenuation with much lower parasitemia in mice as compared to the wild type. Inoculation with mutant Tulahuen provided strong protection against lethal challenge. No obvious differences in the protective effect of TCC wild type versus TCC mutant parasites were observed. Mice challenged with virulent parasites a year after the original infection with the mutant parasites still displayed a significant control over the secondary infection, indicating that immunization with the attenuated T. cruzi confer a strong protective immunity with a prolong immune memory [72].

However, despite the promising data of the attenuated live T. cruzi vaccines mentioned above, these attenuated strains are still not safe human vaccines because of the danger of reversion of an attenuated strain to a virulent form and the increased virulence of attenuated strains in immune compromised individuals. For example, TCC is a naturally attenuated strain of $T$. cruzi which that was initially thought to be unable to persistently infect immune competent mice was found to cause persist infection in animal models [72]. Therefore, it is important to develop an attenuated vaccine which can provide sterile immunity for the hosts. Also, attenuated vaccines should be evaluated in different animal models to overcome the limitation of murine models.

\section{Novel Strategy to Develop Safe and Reliable Vaccines Against Chagas Disease}

Genetic engineering approaches have provided powerful tools for elucidating unknown gene functions and factors involved in gene regulation. Gene targeted replacement by homologous recombination has been one of the most powerful methods for many organisms. This method leads to a potent, safe and well-tolerated Plasmodium falciparum live attenuated vaccine [65]. In T. cruzi, this method is laborious and time-consuming. Homologous recombination in T. cruzi has a very low efficiency and the drug selection process is slow as compared to other kinetoplastids. As a result, only a few mutant strains of T. cruzi have been evaluated as live attenuated vaccines.

Recently, the CRISPR-Cas9 system has been established in T. cruzi [73]. This system has enabled rapid, targeted modifications of wide range of genomes [73]. The system has proven to be relative ease and high efficiency, and has the ability to achieve multiple modifications in T. cruzi. With this system, more genes will be disrupted in T. cruzi and genes that mediate stage differentiation will be discovered, which can facilitate the development of vaccine strains. For example, a strain of $T$. cruzi, which can only infect a host but cannot differentiate to another form, will not be able to cause persistent infection in a host and could be used as a live attenuated vaccine to induce protective immunity. To obtain such a strain, new techniques for inducible gene expression in T. cruzi are necessary. Our laboratory has recently established techniques that should prove useful for these studies.

Rapid regulation of protein levels can be readily achieved in T. cruzi, by expression of a target protein as a fusion with the destabilization domain (ddFKBP) of the "rapamycin binding protein". In the absence of the synthetic legend, Shield-1, proteins bearing this domain are rapidly degraded by the cytoplasmic proteosome. However, protein stabilization can be rapidly achieved by treatment with and reversible binding of Shield-1 to ddFKBP [74]. This method can be used to make essential gene disruption in T. cruzi feasible by expressing a copy of a same gene with an inducible form by adding a ddFKBP domain. For example, if an essential gene in T. cruzi is knocked out in presence of inducible copy of the same gene, withdrawing the expression of the inducible expression will cause the mutant T. cruzi to die. This type of mutant can be used to immunize the animals and induce sufficient immunity without posing a risk.

We have also established another effective inducible system for T. cruzi employing the degradation domain based on the escherichia coli Dihydrofolate Reductase (ecDHFR). The DHFR Degradation Domain (DDD) can be stabilized by trimethoprim-lactate and can be used to express detrimental or toxic proteins. T. cruzi lines with Alpha-toxin, Cecropin A and GFP under the control of DDD with a Hem Agglutinin tag (HA) were developed. Interestingly, amastigotes bearing GFP- DDDHA, Alpha-toxin-DDDHA, Cecropin A-DDDHA and DDDHA all resulted in inducible cell death with these fusions, indicating that DDDHA protein is also detrimental to amastigotes [75]. These strains were found to be attenuated in mouse experiments and infection of mice with these strains provided significant protection against lethal infection with wild type strains. Potentially, these strains may be used as vaccine strains against T. cruzi infections and the DDD technique can serve as a bio-safety device to eliminate parasitism. The combination of this approach with other methods such as gene deletion should significantly improve the safety of an attenuated live T. cruzi vaccine because we can terminate parasitism in the immunized host by turning on the bio-safety device.

\section{Conclusion}

Development of a vaccine against Chagas disease has been delayed due to controversy about the pathogenic mechanisms that underlie disease progression and concerns about the role of autoimmunity in the development of chronic Chagas disease. The genetic complexity of T. cruzi and the lack for efficient engineering techniques for gene disruption have also hindered progress in the generation of suitable strains for vaccines. It is clear, however, that prophylactic vaccines will be cost effective strategies for preventing the disease and for controlling the domestic reservoir of T. cruzi. Therapeutic vaccines could also play a significant role in increasing treatment efficacy or delaying disease progression.

Recombinant subunit vaccines based on T. cruzi antigens are easy to produce and safe. It is convenient to have a variety of combinations of these antigens to improve the stimulation of immune system. However, this type of experimental vaccine has not demonstrated robust protection against T. cruzi infection. The history of the development of Malaria subunit vaccines may serve a guide in the outcome of T. cruzi subunit vaccines. The most advanced subunit malaria vaccine, RTS, S/AS01 only reduced the rates of clinical and severe malaria acquired over a 12 -month period by 31.3 and $36.6 \%$, respectively [65]. However, T. cruzi recombinant subunit vaccines may be used as therapeutic vaccines to improve drug treatment outcome due to stimulation of host immune responses. DNA vaccines expressing T. cruzi antigens are more likely to stimulate Th1 helper $\mathrm{T}$ cells and IFN- $\gamma$ secretion which are necessary to confer protection against T. cruzi infection. However, the protection from this approach is still not robust and the persistent of T. cruzi infection in tissue has not been prevented with the DNA subunit vaccines. In addition, there are concerns about the safety with this approach. With the development of new vector system and T. cruzi antigens, one day this approach may be useful.

Recently, the success of a Malaria sporozoite vaccine demonstrated a novel approach to produce an attenuated live vaccine [65]. With the advent of inducible protein expression methods and inducible suicide vector systems as well as the CRISPR-Cas9 system, live attenuated 
T. cruzi strains as veterinary and human vaccines will be possible in the near future and hold significant promise as vaccines for both prophylaxis and therapy.

\section{Acknowledgments}

This work was supported by NIH grant AI-103450 to H Huang.

\section{References}

1. [No authors listed] (2015) Chagas disease in Latin America: an epidemiological update based on 2010 estimates. Wkly Epidemiol Rec 90: 33-43.

2. Rassi A Jr, Rassi A, Marin-Neto JA (2010) Chagas disease. Lancet 375: 1388-1402.

3. Hotez PJ, Alvarado M, Basáñez MG, Bolliger I, Bourne R, et al. (2014) The global burden of disease study 2010: interpretation and implications for the neglected tropical diseases. PLoS Negl Trop Dis 8: 2865.

4. Lee BY, Bacon KM, Bottazzi ME, Hotez PJ (2013) Global economic burden of Chagas disease: a computational simulation model. Lancet Infect Dis 13 : $342-348$.

5. Alarcón de Noya B, Díaz-Bello Z, Colmenares C, Ruiz-Guevara R, Mauriello L, et al. (2010) Large urban outbreak of orally acquired acute Chagas disease at a school in Caracas, Venezuela. J Infect Dis 201: 1308-1315.

6. Wendel S (2010) Transfusion transmitted Chagas disease: is it really under control? Acta Trop 115: 28-34.

7. Tanowitz HB, Machado FS, Jelicks LA, Shirani J, de Carvalho AC, et al. (2009) Perspectives on Trypanosoma cruzi-induced heart disease (Chagas disease). Prog Cardiovasc Dis 51: 524-539.

8. Bern C, Montgomery SP, Herwaldt BL, Rassi A Jr, Marin-Neto JA, et al. (2007) Evaluation and treatment of chagas disease in the United States: a systematic review. JAMA 298: 2171-2181.

9. Garcia S, Ramos CO, Senra JF, Vilas-Boas F, Rodrigues MM, et al. (2005) Treatment with benznidazole during the chronic phase of experimental Chagas' disease decreases cardiac alterations. Antimicrob Agents Chemother 49: $1521-1528$.

10. Morillo CA, Marin-Neto JA, Avezum A, Sosa-Estani S, Rassi A Jr, et al. (2015) Randomized Trial of Benznidazole for Chronic Chagas' Cardiomyopathy. N Engl J Med 373: 1295-1306.

11. Zerba EN (1999) Susceptibility and resistance to insecticides of Chagas disease vectors. Medicina (B Aires) 59: 41-46.

12. Lee BY, Bacon KM, Connor DL, Willig AM, Bailey RR (2010) The potentia economic value of a Trypanosoma cruzi (Chagas disease) vaccine in Latin America. PLoS Negl Trop Dis 4: 916.

13. Ouaissi A, Guilvard E, Delneste Y, Caron G, Magistrelli G, et al. (2002) The Trypanosoma cruzi Tc52-released protein induces human dendritic cell maturation, signals via Toll-like receptor 2 , and confers protection against lethal infection. J Immunol 168: 6366-6374.

14. Bafica A, Santiago HC, Goldszmid R, Ropert C, Gazzinelli RT, et al. (2006) Cutting edge: TLR9 and TLR2 signaling together account for MyD88-dependent control of parasitemia in Trypanosoma cruzi infection. J Immunol 177: 3515-3519.

15. Maganto-Garcia E, Punzon C, Terhorst C, Fresno M (2008) Rab5 activation by Toll-like receptor 2 is required for Trypanosoma cruzi internalization and replication in macrophages. Traffic 9: 1299-1315.

16. Caetano BC, Carmo BB, Melo MB, Cerny A, dos Santos SL, et al. (2011) Requirement of UNC93B1 reveals a critical role for TLR7 in host resistance to primary infection with Trypanosoma cruzi. J Immunol 187: 1903-1911.

17. Oliveira AC, de Alencar BC, Tzelepis F, Klezewsky W, da Silva RN, et al. (2010) Impaired innate immunity in TIr4(-/-) mice but preserved CD8+ T cell responses against Trypanosoma cruzi in TIr4-, TIr2-, TIr9- or Myd88-deficient mice. PLoS Pathog 6: 1000870 .
18. Silva GK, Gutierrez FR, Guedes PM, Horta CV, Cunha LD, et al. (2010) Cutting edge: nucleotide-binding oligomerization domain 1-dependent responses account for murine resistance against Trypanosoma cruzi infection. J Immunol 184: 1148-1152.

19. Vespa GN, Cunha FQ, Silva JS (1994) Nitric oxide is involved in control of Trypanosoma cruzi-induced parasitemia and directly kills the parasite in vitro. Infect Immun 62: 5177-5182.

20. Sardinha LR, Elias RM, Mosca T, Bastos KR, Marinho CR, et al. (2006) Contribution of NK, NK T, gamma delta $T$, and alpha beta $T$ cells to the gamma interferon response required for liver protection against Trypanosoma cruzi. Infect Immun. 74: 2031-2042.

21. Tzelepis F, de Alencar BC, Penido ML, Gazzinelli RT, Persechini PM, et al. (2006) Distinct kinetics of effector CD8+ cytotoxic T cells after infection with Trypanosoma cruzi in naive or vaccinated mice. Infect Immun 74: 2477-2481.

22. Kurup SP, Tarleton RL (2013) Perpetual expression of PAMPs necessary for optimal immune control and clearance of a persistent pathogen. Nat Commun 4: 2616.

23. Poncini CV, Alba Soto CD, Batalla E, Solana ME, González Cappa SM (2008) Trypanosoma cruzi induces regulatory dendritic cells in vitro. Infect Immun 76: 2633-2641.

24. Vasconcelos JR, Bruña-Romero O, Araújo AF, Dominguez MR, Ersching J, et al. (2012) Pathogen-induced proapoptotic phenotype and high CD95 (Fas) expression accompany a suboptimal CD8+ T-cell response: reversal by adenoviral vaccine. PLoS Pathog 8: 1002699.

25. Albareda MC, Olivera GC, Laucella SA, Alvarez MG, Fernandez ER, et al. (2009) Chronic human infection with Trypanosoma cruzi drives CD4+ T cells to immune senescence. J Immunol 183: 4103-4108.

26. Leavey JK, Tarleton RL (2003) Cutting edge: dysfunctional CD8+ T cells reside in nonlymphoid tissues during chronic Trypanosoma cruzi infection. J Immunol 170: 2264-2268.

27. Martin DL, Weatherly DB, Laucella SA, Cabinian MA, Crim MT, et al. (2006) CD8+ T-Cell responses to Trypanosoma cruzi are highly focused on strain-variant trans-sialidase epitopes. PLoS Pathog 2: 77.

28. Garg N, Bhatia V (2005) Current status and future prospects for a vaccine against American trypanosomiasis. Expert Rev Vaccines 4: 867-880.

29. Ruiz AM, Esteva M, Cabeza Meckert P, Laguens RP, Segura EL (1985) Protective immunity and pathology induced by inoculation of mice with different subcellular fractions of Trypanosoma cruzi. Acta Trop 42: 299-309.

30. Wrightsman RA, Miller MJ, Saborio JL, Manning JE (1995) Pure paraflagellar rod protein protects mice against Trypanosoma cruzi infection. Infect Immun 63: $122-125$.

31. Miller MJ, Wrightsman RA, Manning JE (1996) Trypanosoma cruzi. protective immunity in mice immunized with paraflagellar rod proteins is associated with a T-helper type 1 response. Exp Parasitol 84: 156-167.

32. Snary D (1983) Cell surface glycoproteins of Trypanosoma cruzi: protective immunity in mice and antibody levels in human chagasic sera. Trans $\mathrm{R}$ Soc Trop Med Hyg 77: 126-129.

33. Araujo FG, Morein B (1991) Immunization with Trypanosoma cruzi epimastigote antigens incorporated into iscoms protects against lethal challenge in mice. Infect Immun 59: 2909-2914.

34. Gomes YM, Abath FG, Nakazawa M, Minoprio P, Vouldoukis I, et al. (1999) Partial protection of mice against Trypanosoma cruzi after immunizing with the TcY 72 antigenic preparation. Mem Inst Oswaldo Cruz 94: 167-172.

35. Taibi A, Plumas-Marty B, Guevara-Espinoza A, Schöneck R, Pessoa H, et al. (1993) Trypanosoma cruzi. immunity-induced in mice and rats by trypomastigote excretory-secretory antigens and identification of a peptide sequence containing a T cell epitope with protective activity. J Immunol 151: 2676- 2689.

36. Gruppi A, Cerban F, Pistoresi-Palencia MC, Vottero-Cima E (1995) Trypanosoma cruzi. transfer of protection by lymph node cells obtained from mice immunized with exoantigens of pl 4.5. Exp Parasitol 80: 382-389. 
37. Garcia CA, Oliveira EC, Sakurada JK, Santos LM (2000) Protective immunity induced by a Trypanosoma cruzi soluble extract antigen in experimental Chagas' disease. Role of interferon gamma. Immunol Invest 29: 1-12.

38. Pereira VR, Lorena VM, Verçosa AF, Silva ED, Ferreira AG, et al. (2003) Antibody isotype responses in Balb/c mice immunized with the cytoplasmic repetitive antigen and flagellar repetitive antigen of Trypanosoma cruzi. Mem Inst Oswaldo Cruz 98: 823-825

39. Luhrs KA, Fouts DL, Manning JE (2003) Immunization with recombinant paraflagellar rod protein induces protective immunity against Trypanosoma cruzi infection. Vaccine 21: 3058-3069.

40. Vasconcelos JR, Boscardin SB, Hiyane MI, Kinoshita SS, Fujimura AE, et al. (2003) A DNA-priming protein-boosting regimen significantly improves type 1 immune response but not protective immunity to Trypanosoma cruzi infection in a highly susceptible mouse strain. Immunol Cell Biol 81: 121-129.

41. Cazorla SI, Frank FM, Becker PD, Arnaiz M, Mirkin GA, et al. (2010) Redirection of the immune response to the functional catalytic domain of the cystein proteinase cruzipain improves protective immunity against Trypanosoma cruzi infection. J Infect Dis 202: 136-144.

42. Dumonteil E, Bottazzi ME, Zhan B, Heffernan MJ, Jones K, et al. (2012) Accelerating the development of a therapeutic vaccine for human Chagas disease: rationale and prospects. Expert Rev Vaccines 11: 1043-1055.

43. Martinez-Campos V, Martinez-Vega $P$, Ramirez-Sierra MJ, Rosado-Vallado M, Seid CA, et al. (2015) Expression, purification, immunogenicity, and protective efficacy of a recombinant Tc24 antigen as a vaccine against Trypanosoma cruzi infection in mice. Vaccine 33: 4505-4512.

44. Alarcon JB, Waine GW, McManus DP (1999) DNA vaccines: technology and application as anti-parasite and anti-microbial agents. Adv Parasitol 42: 343410.

45. Planelles L, Thomas MC, Alonso C, López MC (2001) DNA immunization with Trypanosoma cruzi HSP70 fused to the KMP11 protein elicits a cytotoxic and humoral immune response against the antigen and leads to protection. Infect Immun 69: 6558-6563.

46. Planelles L, Thomas M, Pulgar M, Marañón C, Grabbe S, et al. (2002) Trypanosoma cruzi heat-shock protein-70 kDa, alone or fused to the parasite KMP11 antigen, induces functional maturation of murine dendritic cells. Immunol Cell Biol 80: 241-247.

47. Morell M, Thomas MC, Caballero T, Alonso C, López MC (2006) The genetic immunization with paraflagellar rod protein-2 fused to the HSP70 confers protection against late Trypanosoma cruzi infection. Vaccine 24: 7046-7055.

48. Qazi KR, Oehlmann W, Singh M, López MC, Fernández C (2007) Microbial heat shock protein 70 stimulatory properties have different TLR requirements. Vaccine 25: 1096-1103.

49. Cuellar A, Santander SP, Thomas Mdel C, Guzmán F, Gómez A, et al. (2008) Monocyte-derived dendritic cells from chagasic patients vs healthy donors secrete differential levels of IL-10 and IL-12 when stimulated with a protein fragment of Trypanosoma cruzi heat-shock protein-70. Immunol Cell Biol 86 : 255-260.

50. Marañón C, Egui A, Carrilero B, Thomas MC, Pinazo MJ, et al. (2011) Identification of HLA-A*02:01-restricted CTL epitopes in Trypanosoma cruzi heat shock protein-70 recognized by Chagas disease patients. Microbes Infect 13: 1025-1032.

51. Flores-García Y, Rosales-Encina JL, Satoskar AR, Talamás-Rohana P (2011) IL-10-IFN-y double producers CD4+ T cells are induced by immunization with an amastigote stage specific derived recombinant protein of Trypanosoma cruzi. Int J Biol Sci 7: 1093-1100.

52. Carabarin-Lima A, González-Vázquez MC, Baylon-Pacheco L, Tsutsumi V Talamás-Rohana $P$, et al. (2011) Immunization with the recombinant surface protein rTcSP2 alone or fused to the CHP or ATPase domain of TcHSP70 induces protection against acute Trypanosoma cruzi infection. J Vaccines Vaccin 1: 3 .
53. Rodríguez-Morales O, Pérez-Leyva MM, Ballinas-Verdugo MA, Carrillo-Sánchez SC, Rosales-Encina JL, et al. (2012) Plasmid DNA immunization with Trypanosoma cruzi genes induces cardiac and clinical protection against Chagas disease in the canine model. Vet Res 43: 79.

54. Rodríguez-Morales O, Carrillo-Sánchez SC, García-Mendoza H, Aranda-Fraustro A, Ballinas-Verdugo MA, et al. (2013) Effect of the plasmid-DNA vaccination on macroscopic and microscopic damage caused by the experimental chronic Trypanosoma cruzi infection in the canine model. Biomed Res Int 2013: 826570 .

55. Gupta S, Garg NJ (2013) TcVac3 induced control of Trypanosoma cruzi infection and chronic myocarditis in mice. PLoS One 8: 59434.

56. Gupta S, Smith C, Auclair S, Delgadillo Ade J, Garg NJ (2015) Therapeutic Efficacy of a Subunit Vaccine in Controlling Chronic Trypanosoma cruzi Infection and Chagas Disease Is Enhanced by Glutathione Peroxidase Over-Expression. PLoS One 10: 0130562

57. Gupta S, Silva TS, Osizugbo JE, Tucker L, Spratt HM, et al. (2014) Serum-mediated activation of macrophages reflects TcVac2 vaccine efficacy against Chagas disease. Infect Immun 82: 1382-1389.

58. Nogueira RT, Nogueira AR, Pereira MC, Rodrigues MM, Neves PC, et al (2013) Recombinant yellow fever viruses elicit CD8+ T cell responses and protective immunity against Trypanosoma cruzi. PLoS One 8: 59347.

59. Quijano-Hernández IA, Castro-Barcena A, Vázquez-Chagoyán JC, Bolio-González ME, Ortega-López J, et al. (2013) Preventive and therapeutic DNA vaccination partially protect dogs against an infectious challenge with Trypanosoma cruzi. Vaccine 31: 2246-2252.

60. Vasconcelos JR, Dominguez MR, Neves RL, Ersching J, Araújo A, et al. (2014) Adenovirus vector-induced CD8+ T effector memory cell differentiation and recirculation, but not proliferation, are important for protective immunity against experimental Trypanosoma cruzi infection. Hum Gene Ther 25: $350-363$

61. Cazorla SI, Matos MN, Cerny N, Ramirez C, Alberti AS, et al. (2015) Oral multicomponent DNA vaccine delivered by attenuated Salmonella elicited immunoprotection against American trypanosomiasis. J Infect Dis 211: 698-707.

62. Cerny N, Alberti AS, Bivona AE, De Marzi MC, Frank FM, et al.(2015) Coadministration of cruzipain and GM-CSF DNAs, a new immunotherapeutic vaccine against Trypanosoma cruzi infection. Hum Vaccin Immunother 27: 0.

63. Pereira IR, Vilar-Pereira G, Marques V, da Silva AA, Caetano B, et al. (2015) A human type 5 adenovirus-based Trypanosoma cruzi therapeutic vaccine re-programs immune response and reverses chronic cardiomyopathy. PLoS Pathog 11: 1004594.

64. Aparicio-Burgos JE, Zepeda-Escobar JA, de Oca-Jimenez RM, Estrada-Franco JG, Barbabosa-Pliego A, et al. (2015) Immune protection against Trypanosoma cruzi induced by TcVac4 in a canine model. PLoS Negl Trop Dis 9: 0003625 .

65. Seder RA, Chang LJ, Enama ME, Zephir KL, Sarwar UN, et al. (2013) Protection against malaria by intravenous immunization with a nonreplicating sporozoite vaccine. Science 341: 1359-1365

66. Lima MT, Rondinelli R, Sarno EN, Barcinski MA, Gattas CR (1986) Preliminary studies on the infection of BALB/c mice with a clone of Trypanosoma cruzi. Mem Inst Oswaldo Cruz 81: 124.

67. Rowland EC, Ritter DM (1984) Corpus Christi strain-induced protection to Trypanosoma cruzi infection in $\mathrm{C} 3 \mathrm{H}(\mathrm{He})$ mice: transfer of resistance to Brazil strain challenge with lymphocytes. J Parasitol 70: 760-766.

68. Barrio AB, Van Voorhis WC, Basombrío MA (2007) Trypanosoma cruzi. attenuation of virulence and protective immunogenicity after monoallelic disruption of the cub gene. Exp Parasitol 117: 382-389.

69. Zago MP, Barrio AB, Cardozo RM, Duffy T, Schijman AG, et al. (2008) Impairment of infectivity and immunoprotective effect of a LYT1 null mutant of Trypanosoma cruzi. Infect Immun 76: 443-451. 
70. Basombrío MA, Gómez L, Padilla AM, Ciaccio M, Nozaki T, et al. (2002) Targeted deletion of the gp72 gene decreases the infectivity of Trypanosoma cruzi for mice and insect vectors. J Parasitol 88: 489-493.

71. Collins MH, Craft JM, Bustamante JM, Tarleton RL (2011) Oral exposure to Trypanosoma cruzi elicits a systemic $\mathrm{CD}^{+} \mathrm{T}$ cell response and protection against heterotopic challenge. Infect Immun 79: 3397-3406.

72. Perez Brandan C, Padilla AM, Xu D, Tarleton RL, Basombrio MA (2011) Knockout of the dhfr-ts gene in Trypanosoma cruzi generates attenuated parasites able to confer protection against a virulent challenge. PLoS Negl Trop Dis 5: 1418
73. Peng D, Kurup S, Yao P, Mining TA, Tarleton RL (2014) CRISPR-Cas9-Mediated Single-Gene and Gene Family Disruption in Trypanosoma cruzi. MBio 6: 02097-02114.

74. Ma YF, Weiss LM, Huang H (2012) A method for rapid regulation of protein expression in Trypanosoma cruzi. Int J Parasitol 42: 33-37.

75. Ma YF, Weiss LM, Huang H (2015) Inducible suicide vector systems for Trypanosoma cruzi. Microbes Infect 17: 440-450. 